\title{
Animal models of venous thrombosis
}

\author{
Hassan Albadawi ${ }^{1}$, Avery A. Witting ${ }^{1}$, Yash Pershad ${ }^{1}$, Alex Wallace ${ }^{2}$, Andrew R. Fleck ${ }^{2}$, Peter Hoang ${ }^{2}$, Ali \\ Khademhosseini, ${ }^{3,4}$, Rahmi Oklu, ${ }^{1,3}$
}

${ }^{1}$ Department of Radiology, Division of Vascular \& Interventional Radiology, ${ }^{2}$ Department of Radiology, Mayo Clinic, Phoenix, AZ, USA; ${ }^{3}$ Biomaterials Innovation Research Center, Brigham and Women's Hospital \& Harvard Medical School, Cambridge, MA, USA; ${ }^{4}$ Harvard-MIT Division of Health Sciences and Technology, Massachusetts Institute of Technology, Cambridge, MA, USA

Contributions: (I) Conception and design: All authors; (II) Administrative support: None; (III) Provision of study materials or patients: None; (IV) Collection and assembly of data: None; (V) Data analysis and interpretation: None; (VI) Manuscript writing: All authors; (VII) Final approval of manuscript: All authors.

Correspondence to: Hassan Albadawi, MD. Department of Radiology, Division of Interventional Radiology, CRB 1-265, Mayo Clinic, 13400 E Shea Blvd, Scottsdale, AZ 85259, USA. Email: Albadawi.hassan@mayo.edu.

\begin{abstract}
Venous thrombosis (VT) is a prevalent clinical condition with significant adverse sequela or mortality. Anticoagulation and pharmacologic or pharmacomechanical thrombolytic therapies are the mainstays of VT treatment. An understanding of thrombosis biology will allow for more effective VTtailored diagnosis and therapy. In vivo models of thrombosis provide indispensable tools to study the pathogenesis of thrombus formation and to evaluate novel therapeutic or preventive adjuncts for VT management or prevention. In this article, we review the most prominent in vivo models of VT created in rodents and swine species and outline how each model can serve as a useful tool to promote our understanding of VT pathogenesis and to examine novel therapies.
\end{abstract}

Keywords: Veins; thrombosis; venous thrombosis (VT); experimental animal models; thrombolytic therapy; blood coagulation

Submitted Jul 12, 2017. Accepted for publication Aug 07, 2017.

doi: $10.21037 / \mathrm{cdt} .2017 .08 .10$

View this article at: http://dx.doi.org/10.21037/cdt.2017.08.10

\section{Introduction}

Venous thrombosis (VT) is a common clinical condition with a significant financial burden on the US healthcare system. Despite its prevalence, VT diagnosis and management remains rudimentary (1-4). The number of cases of VT in the US exceeds 1 million per year, most commonly occurring in the lower extremities $(1,4)$. The etiology of VT is multifactorial, involving risk factors such as genetics, venous endothelial injury (e.g., trauma or surgery), hypercoagulability (cancer, pregnancy, protein $\mathrm{C}$ mutations), and venous stasis (damage to venous valves, immobility, previous thrombus) $(2,5)$. VT can lead to a myriad of complications, including swelling, erythema, neurovascular compromise, tissue necrosis or limb loss, acute respiratory symptoms, pulmonary hypertension, cardiovascular collapse, thromboembolism, and death.
Anticoagulation and thrombolytic therapies are the mainstays of VT treatment despite a rapid decrease in the treatment's effectiveness as chronic thrombus transformation evolves (5). While most cases of VT resolve with treatment, residual fibrotic changes persist in many patients. As a thrombus matures, fibrosis can cause postthrombotic syndrome, leading to chronic pain, heaviness, leg cramping, limb edema, stasis dermatitis, and, at end stage, venous ulcerations $(6,7)$. Due to the dynamic nature of thrombosis, the age and composition of a thrombus are critical to its management $(8,9)$. Venous thrombus results from interactions between the vein wall, inflammatory cells, platelets, and coagulation factors under variable flow conditions in the setting of a constantly changing milieu of oxygen tension, inflammatory mediators, and acidity $(10,11)$. Historically, the Virchow's triad established the principles of thrombogenesis in humans $(12,13)$. The principle is based 
on three elements which consists of: blood stasis (secondary to immobility, congestive heart failure, vein compressions), alteration to a vein wall (secondary to previous thrombosis, vein inflammation or infection, direct vein wall trauma, varicose veins formation), and hypercoagulability state due to antiphospholipid syndrome, hyperhomocysteinaemia, lower-limb surgery or trauma, childbirth, polycythaemia, neoplastic disease, oral contraceptives.

With a better understanding of VT pathogenesis, more effective and precise therapies can be developed (5). Historically, in vivo models of thrombosis have provided essential tools for studying VT pathogenesis and for evaluating novel therapeutic or preventive adjuncts. Investigators have exploited the elements defined by the Virchow's triad to develop animal models of VT, including various techniques to induce vascular wall injury, blood stasis, and activation of the local coagulation process. A plethora of animal models of VT have been created in small and large animal species at different anatomical locations to serve various purposes; however, investigators may not be aware of specific models and their relevance to the human condition. Therefore, it is important to understand the strengths and weaknesses of each model along with the potential of each one to elucidate VT pathogenesis or test novel therapies. Here, we review the benefits and limitations of the most common large and small animal models for studying VT.

\section{Rodents models of VT}

When using rodents in general-and mice in particularfor models of VT, several challenges have discouraged investigators previously from using them to study thrombosis. Regardless of rodent's small body masses and short life spans, they have physiologic and genetic variations which affect thrombogenesis and make recapitulating human pathology difficult. In addition, rodent models of VT are not useful for clinical-like setting of endovascular interventions because of their small size. In contrast, several advantages make rodents useful in laboratory applications. For instance, the short estrus cycle of rodents allows them to reproduce rapidly with low maintenance costs and enables the use of genetically modified strains or human disease models. The large diversity of genetically modified mouse strains has allowed a greater understanding of the blood coagulation cascade and the fibrinolytic system. Furthermore, numerous genes encoding platelet, vascular endothelial, or vascular smooth muscle cell associated proteins have been targeted in mice. These genetically modified animals have been valuable tools for elucidating gene function associated with thrombosis in vivo. While experiments with rodents were previously technically challenging, the development of innovative molecular, surgical, and imaging tools have improved the technical feasibility and standardization for the use of small animals as models of thrombosis. Thrombosis can be induced in rodent's small vessels, such as the mesenteric or femoral veins. However, thrombosis is more commonly induced in the inferior vena cava (IVC) because there the coagulation cascade generates better thrombus size for subsequent analyses and acts as a better platform for chronic thrombosis studies (14). Several animal models were based on venous stasis (15-18), endothelial injury (19-23), or by inducing local hypercoagulability (24-27). Other techniques were based on a divergence of the Wessler's principle, which focuses on studying the pathogenesis of thrombosis and the effect of procoagulant molecules on thrombus growth. Recognizing the variations that exist between these models and their limitations in assessing the pre-thrombotic circumstances that lead to thrombogenesis is extremely important. Listed below are most commonly used models for inducing VT in rodents.

\section{Rodents model of venous stasis}

This model, developed in rats (28-30) and mice (31-35), involves complete and permanent occlusion of a major vein such as the IVC associated with sacrificing of all side branches distal to the left renal vein (Figure 1A,B). The venous stasis reduces venous flow in the IVC, which is verified by measuring proximal venous pressure (36). Many investigators have used this model to study the effect of antithrombotic interventional anticoagulants by measuring thrombus size and resolution. The mechanism of thrombus formation in this model correlates to a combination of venous stasis and increased local release of tissue factor with augmented coagulation inside the vein $(14,37)$. Venous stasis is performed in anesthetized animals followed by a midline laparotomy. The bowels are carefully mobilized outside the abdominal cavity to the animal's left to expose the IVC. The bowls are covered with wet gauze to maintain moisture. Careful blunt dissection exposes the IVC, while sterile saline is applied at regular intervals to the exteriorized bowel to prevent its desiccation. A nonabsorbable 7-0 prolene ligature is looped around the IVC immediately distal to the origin of the left renal vein and then tied, along with all side branches, to completely obstruct blood flow. The posterior branches are cauterized. 

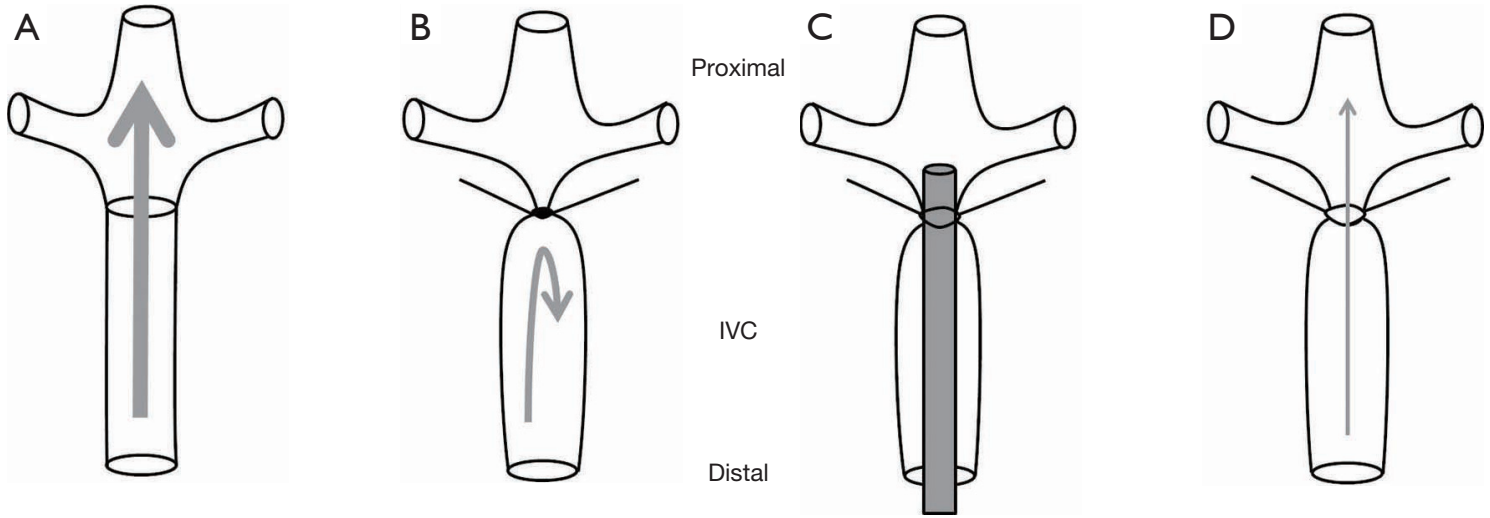

Figure 1 Diagram of venous stasis and stenosis models in the IVC. (A) Normal IVC blood flow; (B) applying a ligature distal to occlude the renal veins creates stasis; (C) placing a spacer on top of the IVC followed by looping a ligature around the IVC and the spacer; (D) subsequently removing the spacer will create venous stenosis with partial IVC occlusion. IVC, inferior vena cava.

Following ligation, the venous tissue can be harvested at variable time points. Ultrasound sonography can be used serially to monitor thrombus progression. The excised venous segment can be used to measure thrombus size and subsequently processed for histologic analysis. This model has high survival rate; hence, it has been useful to assess chronic thrombus organization into a collagen-rich tissue, recanalization, and thrombus resolution. However, the disadvantages of venous occlusion model cannot be reversed and lacks the possibility of re-establishing blood flow by damaging the endothelium. Therefore, venous stasis should only be used to simulate the clinical scenario of complete occlusion. A variation of the venous stasis model was also developed in rats using both complete ligation and induced local thrombosis, as described by Vogel et al. (24). Anesthetized rats are placed in supine position on a heated platform to maintain physiologic temperature. The abdomen wall is entered to expose the IVC. Two ligatures are looped distal to left renal vein around the posterior IVC to form a snare-like configuration. All IVC side branches distal to the left renal vein and proximal to the bifurcation are ligated. Intravascular injection of variable amounts of thrombin $(100-2,000 \mu \mathrm{g} / \mathrm{kg})$ inside the right femoral vein will induce thrombus formation. After $10 \mathrm{~s}$, the two looped snares are tightened around the IVC to induce blood stasis. This model is well-suited for evaluating antithrombotic therapies (38).

\section{Rodent models of venous stenosis}

This model is created in mice that differ from the stasis model by preserving venous blood flow similar to human thrombus without causing vessel wall injury. The mechanism of thrombus formation in the stenosis model is possibly related to "endothelial activation, reduction in blood flow velocity, and disturbed blood flow upstream of the stenosis site" $(39,40)$ (Figure 1C,D). Laparotomy and exteriorization of the intestines are performed under anesthesia before dissecting the IVC from the aorta. A sterile spacer is placed on the exposed IVC followed by passing a 7-0 prolene ligature which loops around the IVC and the spacer. The spacer and the IVC are ligated immediately distal to the renal veins followed by removal of the spacer which will decrease venous blood flow by around 90\% (41). Investigators have used variable spacer sizes of blunt needles. The large variation in the size of the created thrombus and failure to form persistent thrombus in some animals limit the consistency and reliability of this model (14). Certain studies performed the stenosis and permanently ligated all visible side branches using 7-0 prolene suture to ensure that side branches did not influence thrombus size. Highfrequency ultrasound systems can document thrombus size over time after stenosis is induced. However, significant concerns were raised regarding the reported variability in the timing of thrombus size and formation, which limits the ability to document significant differences between groups influenced by side branches (42) or spacer size (39-42). This model is most suited for studying thrombus initiation by platelets interactions and coagulation factors (41). Similarly, this model can also be created in rats $(36,43,44)$. 


\section{Ferric chloride induced venous injury}

In this model, topical application of ferric chloride solution to a surgically exposed venous wall can induce VT due to oxidative damage to the vessel wall $(45,46)$. Depending on the desired timing of thrombus initiation and the extent of thrombus size, a strip of filter paper soaked in selected concentrations of ferric chloride solution is applied to a vein, such as the superficial femoral vein or IVC, for at least 2-3 min. Histologic evaluation will confirm the presence of thrombus. Gustafsson et al. described a modification of this model using ferric-chloride-induced vessel injury combined with stenosis in rats (47). In this model, venous thrombus is induced in partially stenosed IVC followed by local treatment with $10 \%$ ferric chloride solution applied to the external vessel wall for 5 minutes (38). IVC stenosis is performed by looping a cotton ligature around the vessel together with a 20-gauge blunt needle placed next to the IVC distal to the femoral vein. Afterwards, the needle is removed. The ferric chloride via a saturated strip of filter paper is then applied to the external surface of the IVC distal to the stenosis site for 5 minutes. During that 5 minutes period, the exposed tissues are kept hydrated, while the wound is temporarily closed with clamps. One hour after removal of the filter paper, all the side branches of the ligated and treated vessel segment are permanently ligated. This model is mainly used for studying acute thrombus formation since redox chemical stimuli does not simulate deep vein thrombosis (DVT) in humans.

\section{Mouse model of photochemical vein injury}

This model was created in the jugular or the femoral veins in mice (48). Anesthetized mice are placed in the supine position under a surgical microscope. Cervical incision is created to expose and dissect the right internal jugular vein. A Doppler flow probe will be used to assess vessel patency and blood flow. Diluted rose bengal (RB) in phosphatebuffered solution is then systemically administered through a tail vein injection. Immediately before RB injection, a $1.5 \mathrm{~mW}$ green light laser $(540 \mathrm{~nm})$ is shined towards the designated site of injury until thrombus is formed. One of the advantages of this model is rapid thrombus formation with preserved arterial patency. However, RB can potentially be toxic to animals.

\section{Mechanical injury model in mice}

This model was described by Pierangeli et al. that consist of applying forceps to deliver a standardized "pinch" of $1,500 \mathrm{~g} / \mathrm{mm}^{2}$ to a surgically exposed mouse femoral vein $(49,50)$, which result in non-occlusive thrombus formation. The thrombus areas as well as the times of formation and resolution can be recorded. Fiber-optic device transilluminates the injured vein while a video-equipped microscope system is used to visualize the venous injury site. Image analysis can document thrombus size and progression over time. This model is useful to measure kinetics of thrombus growth and expansion, but requires specialized setup and equipment.

\section{Electrolytic thrombosis model of IVC in mice}

The electrolytic method was created in mice as an alternative to the venous stenosis model in order to create a non-occlusive VT. Cooley et al. described this model using transient direct-current electrical injury to induce thrombosis in the common femoral vein of adult mice $(51,52)$. Recently, a variation of the electrolytic stimulation model was created by delivering a current inside the caudal end of the IVC in mice (53-55) (Figure 2). Following IVC exposure, all visible side branches should be ligated using 7-0 prolene ligature while keeping the posterior branches patent. Silver-coated copper wire is inserted into the subcutaneous tissue to create a cathode lead and then is connected to a 25 -gauge needle. The needle is inserted to the caudal IVC to create an anode lead which will deliver a $250 \mu \mathrm{A}$ current over 15 minutes. The current induces free radicals in the venous wall which will subsequently activate the endothelial cells in the IVC. The IVC electrolytic model stimulates thrombosis without thermal effect that maintains blood flow inside the vein resembling the clinical scenario. Investigators reported that this model will reliably result in consistent thrombus size over variable time points following the procedure. Consistent thrombus size will enhance the study of anti-thrombotic and thrombolytic therapy in a more clinically relevant pathology. Moreover, this model can investigate both the acute and chronic stages of VT. However, drawbacks of using this model include prolonged procedural time and potential damage to the venous wall at the needle access site.

\section{Porcine models of DVT}

Many models of VT were created in pigs to study thrombosis using different endovascular or open surgical techniques within different vascular beds (56-60). 

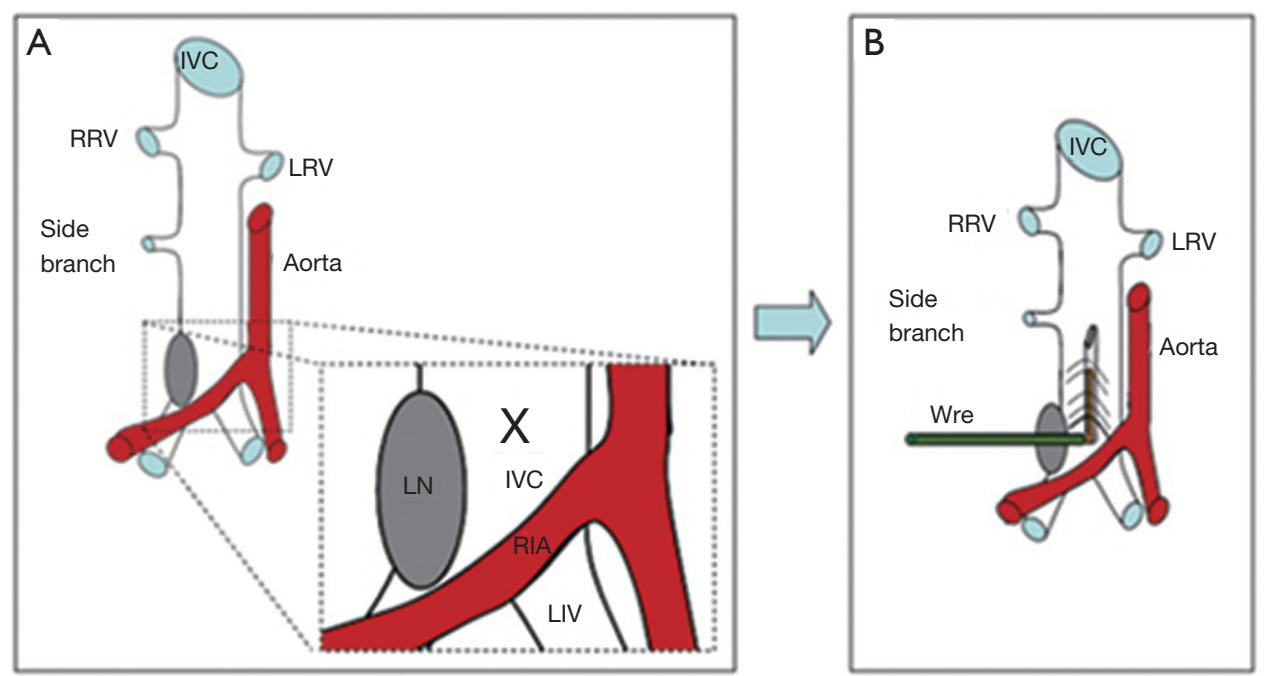

Figure 2 Electrolytic thrombosis model of IVC. (A) Schematic representation of using electrolytic stimulation to induce VT in the IVC in mice as described by Diaz et al. (55); (B) a 25-gauge stainless-steel needle, attached to a silver coated copper wire (KY-30-1-GRN, Electrospec, Dover, NJ, USA) is inserted. RRV, right renal vein; LRV, left renal vein; RIA, right innominate artery; LIV, left innominate vein; LN, lymph node; VT, venous thrombosis; IVC, inferior vena cava.

The development of minimally invasive endovascular interventions created the need for a preclinical animal model of VT that allows for testing of novel therapies. Porcine models have a similar coagulation cascade to humans, and the anatomy of the vascular network in pigs and the human lower extremities is comparable. These similarities allow for surgical or endovascular interventions which simulate the clinical settings. Optimally, thrombus generated in swine models can closely resemble the morphology and cellular composition observed in human thrombus.

\section{Stent-graft model of chronic DVT in swine}

Lin $e t$ al. described a reproducible porcine model of VT to evaluate thrombolysis therapy (61). The superficial femoral veins are exposed through bilateral groin cut downs. A $14 \mathrm{~F}$ introducer sheath is inserted bilaterally in the superficial femoral veins. As described by Lin et al., an endovascular device is created from "tapered polytetrafluoroethylene graft attached within a self-expanding nitinol stent" and delivered to both common iliac veins (61). The tapered graft is inverted within the stent, and the stent-graft is deployed in the common iliac vein, which produces venous thrombus due to venous stasis. A filter is placed in the vena cava to prevent pulmonary embolism. Using this model, after catheter-directed pulse-spray thrombolysis with urokinase and heparin at different time points followed thrombus creation, thrombus resolution showed time-dependent decreases in efficacy with increased thrombus age. Thrombolysis is not effectively passed within 14 days, indicating thrombus transformation to a refractory chronic state (61). A histologic assessment of thrombus morphology was consistent with other models of venous stasis.

\section{Acute DVT induced by thrombin in swine}

Roy et al. described a minimally invasive model of acute VT in the external iliac vein using a balloon catheter to occlude the vein followed by distal trans-catheter infusion of variable doses of thrombin through a microcatheter. Saline was infused on the contralateral side as the control (62). Thrombin infusion was suggested to ensure consistent thrombus formation, since earlier attempts to induce thrombus did not provide consistent results $(63,64)$. In this model, venous flow is maintained at a minimal rate, which is important to create platelet-rich, rather than fibrinrich, thrombus to simulate the human scenario. Similar approaches were used to induce thrombosis in the deep iliac vein associated with thrombin infusion distally to the occlusion site (57). X-ray fluoroscopy, can be used to introduce balloon catheterization and venography can be performed to visualize the venous anatomy. The balloon 


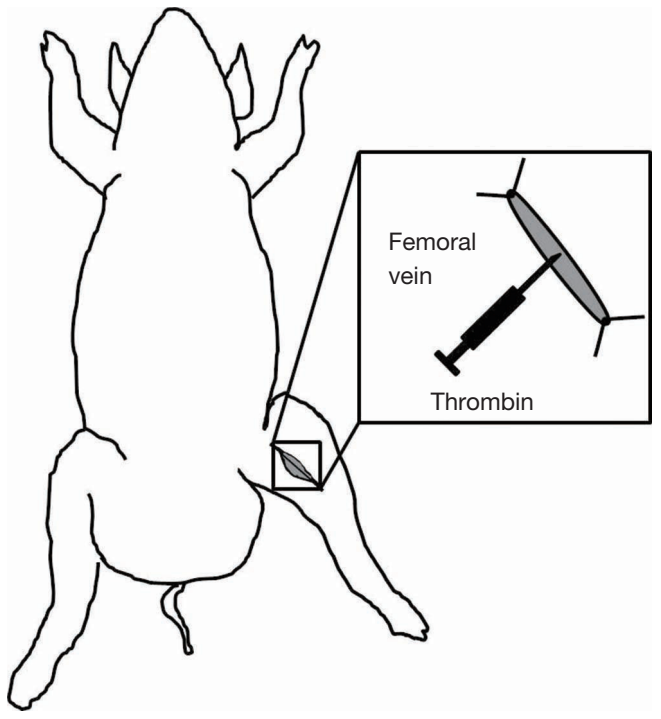

Figure 3 Porcine model of acute femoral vein thrombosis induced by thrombin. Schematic of surgically exposed and isolated femoral vein segment with proximal and distal ligatures applied. Following vein segment isolation, local thrombosis is induced by intraluminal injection of $250 \mathrm{U}$ of thrombin using a 25 -gauge needle. Once thrombus formation is verified, the ligature can be removed.

catheter is navigated through a jugular vein cut down, and an ${ }^{18} \mathrm{~F}$ introducer sheath is delivered over a 0.035 inch $\mathrm{J}$-shaped guide wire. The balloon catheter is inflated just proximal to an identified venous segment that has a minimal collateral presence in order to create adequate stasis, which can enhance subsequent thrombus formation. Once the balloon is in place, thrombin solution is slowly injected, distally past the balloon through the catheter to induce local coagulation. One hour after thrombin injection, the balloon is deflated and removed. MRI and venography can be used to document and characterize the newly formed thrombus. This model is suitable to evaluate novel thrombolytic treatments in a preclinical setting. Using this model, investigators performed thrombectomy with a Fogarty catheter, a percutaneous catheter-based thrombolytic device, and an electrical discharge-induced shock wave. Wang et al. used a similar method to create thrombosis in the superior sagittal sinus, using endovascular access and an occlusion balloon (65). Using a similar method, Maxwell et al. created acute VT in the femoral vein to study the pulsed ultrasound cavitation therapy in juvenile pigs (59). Geier et al. performed laparoscopic ligation of the infra renal vena cava in combination with infusion of thrombin in the femoral vein in pigs to induce chronic VT (60). The resulting thrombi are comparable to human VT in terms of extent, size, and organization process. Minko et al. used this model to test "the efficacy and safety of mechanical thrombectomy for iliac VT using Rotarex and Aspirex catheters in pigs" (66). These investigators used digital subtraction angiography and CT scanning to assess the effectiveness of mechanical thrombectomy. Such imaging techniques can rule out local complications or the presence of pulmonary embolism, as seen in clinical practices.

Robinson et al. developed a similar technique to induce acute $\mathrm{VT}$ in the femoral vein (67). This method involves local injection of thrombin inside an isolated femoral vein segment and partial ligation of the proximal end of the femoral vein segment in swine (Figure 3). The femoral vein dissection is performed followed by careful ligation of the posterior penetrating branches. A $6 \mathrm{~cm}$ vein segment is exposed and dissected followed by applying two ligatures placed distally and proximally to the exposed segment. To induce intravenous coagulation, $250 \mathrm{U}$ of thrombin is injected inside the isolated venous segment using a 25 -gauge needle. Clot formation can be verified by palpating the isolated venous segment. Once thrombus formation is confirmed, the two ligatures can be released. Investigators have used this model to determine "the response of the latex D-dimer signal to acute in situ VT and the minimum dose of exogenous bolus tissue plasminogen activator (t-PA) required to significantly elevate the D-dimer signal" (67).

\section{Swine model of thrombotic caval occlusion}

This model was created to induce thrombosis in the IVC which is created by autologous thrombus injection using the assistance of an intra-caval net knitting trap (Figure 4). The following description of this model was based on a published report by Shi et al. (68). The abdomen wall is surgically opened to expose and isolate the IVC. Two vessel clips are placed on the IVC just distal to the renal veins and proximal to the bifurcation of the common iliac veins, temporarily blocking the IVC. Four 4-0 size polypropylene sutures attached to needles are passed through the IVC wall at anterior, posterior, transverse, and oblique directions $2 \mathrm{~cm}$ below the renal veins. The sutures are then tied consecutively at the outside wall of IVC. The intra-caval net, four sutures intercrossed at the center of the IVC lumen, captures the thrombus forming distally to the created kitting trap. The vessel clips are removed, and the abdomen is closed. An autologous thrombus is prepared and delivered with a $6 \mathrm{~F}$ sheath into the IVC. This sheath was delivered 


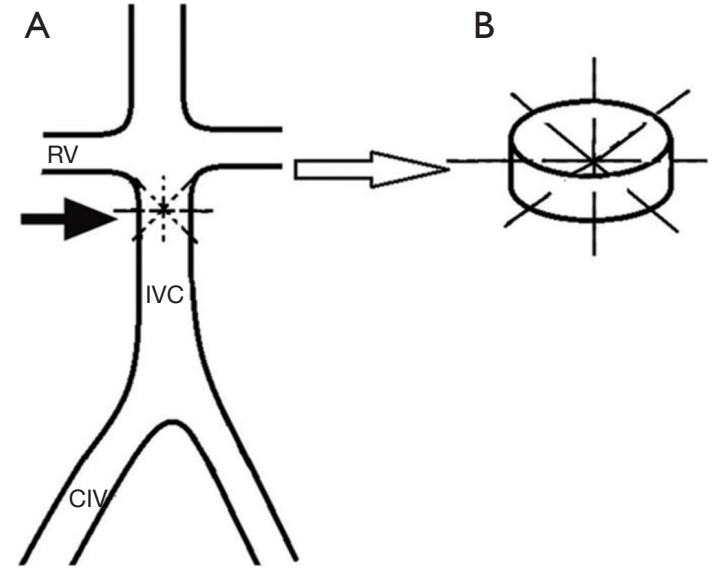

Figure 4 Swine model of thrombotic caval occlusion. (A) Representation of IVC net creation to induce VT aided by autologous thrombus injection as described by Shi et al. (68) autologous. Four sutures are passed through infrarenal IVC at different directions to create intraluminal net (arrow); (B) local visualization of the net. The intra-caval net consisted of four sutures that intercrossed each other at the center of the IVC lumen. RV, renal vein; IVC, inferior vena cava; CIV, common iliac vein; VT, venous thrombosis.

through the femoral vein, having the end placed inside the common iliac vein. The side tube of the sheath connects to the syringe. After injection, the thrombus mainly distributes into the infra-renal IVC trapped by the knitted net. The thrombus size can be evaluated immediately after injection, with the assumption of a cylindrical thrombus formation. Using angiography, investigators demonstrated partial IVC patency at 7 days after the procedure and complete occlusion with its associated collateral vessels at 14 days. This VT model is suitable for interrogating the interaction between the thrombus and the vessel wall along with testing various endovascular devices in a preclinical setting. However, the disadvantage of this model is that it is both invasive and technically challenging.

\section{Discussion}

Animal models of VT were created to depict human DVT and dissect various parameters of complex physiologic, molecular, and cellular contributors to thrombogenesis (12). Realistically, reproducing all components of thrombus microenvironments in vitro to yield translational information is extremely challenging, so each VT model has certain advantages and limitations, along with ideal conditions the model can simulate. Many models involve triggering thrombus formation within a normal vessel bed, which is unlike VT development in humans. In humans, VT usually develops under diseased conditions and utilizes non-pathologic stimuli, so models in normal vessel beds do not recapitulate the stimuli implicated in human VT. Moreover, these methods can potentially alter the vascular bed structure or modify the microenvironment by mechanical, chemical, or thermal effects that do not reflect venous thrombogenesis in humans, thereby limiting their translational benefits. It is also important to recognize significant differences in hemodynamics, vessel size, and coagulation processes between animals and humans, and translate such differences in hemostatic or thrombolysis potential (69). Furthermore, optimizing the study design to include more realistic endpoints and time periods will enhance the ability to detect significant changes following genetic or therapeutic manipulations. Accordingly, no animal model can thoroughly replicate human pathology; however, the existing in vivo models were useful to confirm the efficacy anticoagulation therapy that has shown great potential in vitro. Despite these limitations, rodents became the predominant models to study several aspects of thrombogenesis and test novel therapies because of the availability of molecular tools and the possibility of genetic manipulation. Histologic evaluation of murine thrombus revealed structural changes that are similar to a human venous thrombus (14). Genetic manipulation of selected coagulation factors or cell types in mice resulted in phenotypic changes that mirrored those observed in patients $(40,41,70)$. In addition, anticoagulant and antiplatelet drugs that are used clinically were proven to be effective in mice $(69,71)$, indicating that the mechanisms of thrombus initiation and propagation in rodent models are similar to those in humans. These observations support the use of laboratory mice to study human VT. Furthermore, VT models were useful in titrating dosimetry, potency of thrombolytic agents, or providing preclinical predictive therapeutic value. However, we should be cautious to interpret the results of dose optimization of different antithrombotic strategies using the animal models of VT. Outcome discrepancies create considerable variations from the ultimate dosages appropriate for humans. Continual improvement of the methodology used to study thrombosis in animals is essential. From a technical perspective, further development of practical, relatively inexpensive methods for quantitatively measuring the rate of clot growth is extremely useful. Moreover, the ability to determine the 
kinetics of clot growth and lysis, as opposed to the simpler endpoints of occlusion, will enable more precise and sensitive experiments. It is equally important to develop more clinically relevant thrombosis models, a process that can be achieved by molecular genetic approaches. Each of the described models carries variable advantages or disadvantages and specific features when it comes to model selection. Large animals more closely resemble thrombosis in humans; therefore, they are more suitable for studying various aspects of thrombogenesis and testing novel therapeutic or diagnostic tools compared to smaller species. They allow for translational studies and utilize many available imaging techniques. The pig models are useful for non-invasive assessments of thrombus transformation, using ultrasound elastography (56) and clinically used devices and techniques. Conversely, rodent models of VT have been extensively utilized, because they reproduce rapidly with low maintenance costs and provide availability of genetically modified strains or human disease models. Furthermore, the development of innovative molecular, surgical, and imaging tools will improved the technical feasibility and standardization when using small animal models of VT. Therefore, investigators should take into consideration many factors that will best serve their specific aims when selecting DVT models. These include animal species, setup cost, the need for specialized equipment, technical challenges, and the pathophysiologic factors of the study.

\section{Acknowledgements}

R Oklu acknowledges support from National Institutes of Health (HL137193, EB24403, EB021148, CA172738) and Mayo Clinic.

\section{Footnote}

Conflicts of Interest: The authors have no conflicts of interest to declare.

\section{References}

1. Heit JA. The epidemiology of venous thromboembolism in the community. Arterioscler Thromb Vasc Biol 2008;28:370-2.

2. Morris TA. Natural history of venous thromboembolism. Crit Care Clin 2011;27:869-84.

3. Spyropoulos AC, Lin J. Direct medical costs of venous thromboembolism and subsequent hospital readmission rates: an administrative claims analysis from 30 managed care organizations. J Manag Care Pharm 2007;13:475-86.

4. White RH. The epidemiology of venous thromboembolism. Circulation 2003;107:I4-8.

5. Stanford SN, Sabra A, D'Silva L, et al. The changes in clot microstructure in patients with ischaemic stroke and the effects of therapeutic intervention: a prospective observational study. BMC Neurol 2015;15:35.

6. Henke PK, Comerota AJ. An update on etiology, prevention, and therapy of postthrombotic syndrome. J Vasc Surg 2011;53:500-9.

7. Kahn SR, Shrier I, Julian JA, et al. Determinants and time course of the postthrombotic syndrome after acute deep venous thrombosis. Ann Intern Med 2008;149:698-707.

8. Hillis C, Crowther MA. Acute phase treatment of VTE: Anticoagulation, including non-vitamin $\mathrm{K}$ antagonist oral anticoagulants. Thromb Haemost 2015;113:1193-202.

9. Oklu R, Wicky S. Catheter-directed thrombolysis of deep venous thrombosis. Semin Thromb Hemost 2013;39:446-51.

10. Diamond SL. Systems Analysis of Thrombus Formation. Circ Res 2016;118:1348-62.

11. Henke PK, Wakefield T. Thrombus resolution and vein wall injury: dependence on chemokines and leukocytes. Thromb Res 2009;123 Suppl 4:S72-8.

12. Byrnes JR, Wolberg AS. New findings on venous thrombogenesis. Hamostaseologie 2017;37:25-35.

13. Keller K, Prochaska JH, Coldewey M, et al. History of deep vein thrombosis is a discriminator for concomitant atrial fibrillation in pulmonary embolism. Thromb Res 2015;136:899-906.

14. Diaz JA, Obi AT, Myers DD Jr, et al. Critical review of mouse models of venous thrombosis. Arterioscler Thromb Vasc Biol 2012;32:556-62.

15. Bernat A, Vallée E, Maffrand JP, et al. Antithrombotic effect of ticlopidine in a platelet-independent model of venous thrombosis. Thromb Res 1985;37:279-85.

16. Doutremepuich C, Gestreau JL, Maury MO, et al. Experimental venous thrombosis in rats treated with heparin and a low molecular weight heparin fraction. Haemostasis 1983;13:109-12.

17. Reyers I, Mussoni L, Donati MB, et al. Failure of aspirin at different doses to modify experimental thrombosis in rats. Thromb Res 1980;18:669-74.

18. Thomas DP, Merton RE, Hockley DJ. The effect of stasis on the venous endothelium: an ultrastructural study. $\mathrm{Br} \mathrm{J}$ Haematol 1983;55:113-22.

19. Hladovec J. A sensitive model of venous thrombosis in rats. Thromb Res 1986;43:539-44. 
20. Pescador R, Porta R, Conz A, et al. A quantitative venous thrombosis model with stasis based on vascular lesion. Thromb Res 1989;53:197-201.

21. Solis MM, Cook C, Cook J, et al. Intravenous recombinant soluble human thrombomodulin prevents venous thrombosis in a rat model. J Vasc Surg 1991;14:599-604.

22. Spokas EG, Wun TC. Venous thrombosis produced in the vena cava of rabbits by vascular damage and stasis. J Pharmacol Toxicol Methods 1992;27:225-32.

23. Thomas DP, Merton RE, Wood RD, et al. The relationship between vessel wall injury and venous thrombosis: an experimental study. Br J Haematol 1985;59:449-57.

24. Vogel GM, Meuleman DG, Bourgondiën FG, et al. Comparison of two experimental thrombosis models in rats effects of four glycosaminoglycans. Thromb Res 1989;54:399-410.

25. Wessler S. Thrombosis in the presence of vascular stasis. Am J Med 1962;33:648-66.

26. Wessler S, Gitel SN, Bank H, et al. An assay of the antithrombotic action of warfarin: its correlation with the inhibition of stasis thrombosis in rabbits. Thromb Haemost 1979;40:486-98.

27. Herbert JM, Bernat A, Maffrand JP. Importance of platelets in experimental venous thrombosis in the rat. Blood 1992;80:2281-6.

28. Myers DD Jr, Henke PK, Wrobleski SK, et al. P-selectin inhibition enhances thrombus resolution and decreases vein wall fibrosis in a rat model. J Vasc Surg 2002;36:928-38.

29. Dewyer NA, Sood V, Lynch EM, et al. Plasmin inhibition increases MMP-9 activity and decreases vein wall stiffness during venous thrombosis resolution. J Surg Res 2007;142:357-63.

30. Henke PK, Varma MR, Deatrick KB, et al. Neutrophils modulate post-thrombotic vein wall remodeling but not thrombus neovascularization. Thromb Haemost 2006;95:272-81.

31. Myers DD, Hawley AE, Farris DM, et al. P-selectin and leukocyte microparticles are associated with venous thrombogenesis. J Vasc Surg 2003;38:1075-89.

32. Myers D Jr, Farris D, Hawley A, et al. Selectins influence thrombosis in a mouse model of experimental deep venous thrombosis. J Surg Res 2002;108:212-21.

33. Wojcik BM, Wrobleski SK, Hawley AE, et al. Interleukin-6: a potential target for post-thrombotic syndrome. Ann Vasc Surg 2011;25:229-39.

34. Diaz JA, Ballard-Lipka NE, Farris DM, et al. Impaired fibrinolytic system in ApoE gene-deleted mice with hyperlipidemia augments deep vein thrombosis. J Vasc Surg 2012;55:815-22.

35. Angelillo-Scherrer A, de Frutos P, Aparicio C, et al. Deficiency or inhibition of Gas6 causes platelet dysfunction and protects mice against thrombosis. Nat Med 2001;7:215-21.

36. Pottier P, Planchon B, Truchaud F, et al. Development of an experimental model of pre-thrombosis in rats based on Wessler's principle using a calibrated venous stasis. Blood Coagul Fibrinolysis 2003;14:3-9.

37. Zhou J, May L, Liao P, et al. Inferior vena cava ligation rapidly induces tissue factor expression and venous thrombosis in rats. Arterioscler Thromb Vasc Biol 2009;29:863-9.

38. Peternel L, Drevensek G, Cerne M, et al. Evaluation of two experimental venous thrombosis models in the rat. Thromb Res 2005;115:527-34.

39. Brill A, Fuchs TA, Savchenko AS, et al. Neutrophil extracellular traps promote deep vein thrombosis in mice. J Thromb Haemost 2012;10:136-44.

40. von Brühl ML, Stark K, Steinhart A, et al. Monocytes, neutrophils, and platelets cooperate to initiate and propagate venous thrombosis in mice in vivo. J Exp Med 2012;209:819-35.

41. Brill A, Fuchs TA, Chauhan AK, et al. von Willebrand factor-mediated platelet adhesion is critical for deep vein thrombosis in mouse models. Blood 2011;117:1400-7.

42. Brandt $M$, Schönfelder T, Schwenk $M$, et al. Deep vein thrombus formation induced by flow reduction in mice is determined by venous side branches. Clin Hemorheol Microcirc 2014;56:145-52.

43. McGuinness CL, Humphries J, Waltham M, et al. Recruitment of labelled monocytes by experimental venous thrombi. Thromb Haemost 2001;85:1018-24.

44. Myers DD Jr, Henke PK, Bedard PW, et al. Treatment with an oral small molecule inhibitor of P selectin (PSI697 ) decreases vein wall injury in a rat stenosis model of venous thrombosis. J Vasc Surg 2006;44:625-32.

45. Jaffer FA, Tung CH, Gerszten RE, et al. In vivo imaging of thrombin activity in experimental thrombi with thrombinsensitive near-infrared molecular probe. Arterioscler Thromb Vasc Biol 2002;22:1929-35.

46. Jaffer FA, Tung CH, Wykrzykowska JJ, et al. Molecular imaging of factor XIIIa activity in thrombosis using a novel, near-infrared fluorescent contrast agent that covalently links to thrombi. Circulation 2004;110:170-6.

47. Gustafsson D, Nyström J, Carlsson S, et al. The direct thrombin inhibitor melagatran and its oral prodrug $\mathrm{H}$ 376/95: intestinal absorption properties, biochemical and 
pharmacodynamic effects. Thromb Res 2001;101:171-81.

48. Eitzman DT, Westrick RJ, Nabel EG, et al. Plasminogen activator inhibitor-1 and vitronectin promote vascular thrombosis in mice. Blood 2000;95:577-80.

49. Pierangeli SS, Barker JH, Stikovac D, et al. Effect of human IgG antiphospholipid antibodies on an in vivo thrombosis model in mice. Thromb Haemost 1994;71:670-4.

50. Pierangeli SS, Liu XW, Barker JH, et al. Induction of thrombosis in a mouse model by $\operatorname{IgG}, \operatorname{IgM}$ and $\operatorname{IgA}$ immunoglobulins from patients with the antiphospholipid syndrome. Thromb Haemost 1995;74:1361-7.

51. Cooley BC, Schmeling G. Murine model of large-vein electrolytic injury induction of thrombosis with slow resolution. Thromb Res 2016;140:149-52.

52. Cooley BC, Szema L, Chen CY, et al. A murine model of deep vein thrombosis: characterization and validation in transgenic mice. Thromb Haemost 2005;94:498-503.

53. Diaz JA, Alvarado CM, Wrobleski SK, et al. The electrolytic inferior vena cava model (EIM) to study thrombogenesis and thrombus resolution with continuous blood flow in the mouse. Thromb Haemost 2013;109:1158-69.

54. Diaz JA, Hawley AE, Alvarado CM, et al. Thrombogenesis with continuous blood flow in the inferior vena cava. A novel mouse model. Thromb Haemost 2010;104:366-75.

55. Diaz JA, Wrobleski SK, Hawley AE, et al. Electrolytic inferior vena cava model (EIM) of venous thrombosis. J Vis Exp 2011;(53):e2737.

56. Geier B, Barbera L, Muth-Werthmann D, et al. Ultrasound elastography for the age determination of venous thrombi. Evaluation in an animal model of venous thrombosis. Thromb Haemost 2005;93:368-74.

57. Katoh M, Haage P, Spuentrup E, et al. A porcine deep vein thrombosis model for magnetic resonance-guided monitoring of different thrombectomy procedures. Invest Radiol 2007;42:727-31.

58. Katoh M, Haage P, Wiethoff AJ, et al. Molecular magnetic resonance imaging of deep vein thrombosis using a fibrintargeted contrast agent: a feasibility study. Invest Radiol 2009;44:146-50.

59. Maxwell AD, Owens G, Gurm HS, et al. Noninvasive treatment of deep venous thrombosis using pulsed ultrasound cavitation therapy (histotripsy) in a porcine model. J Vasc Interv Radiol 2011;22:369-77.

60. Geier B, Muth-Werthmann D, Barbera L, et al. Laparoscopic ligation of the infrarenal vena cava in combination with transfemoral thrombin infusion: a new animal model of chronic deep venous thrombosis. Eur J
Vasc Endovasc Surg 2005;29:542-8.

61. Lin PH, Chen C, Surowiec SM, et al. Evaluation of thrombolysis in a porcine model of chronic deep venous thrombosis: an endovascular model. J Vasc Surg 2001;33:621-7.

62. Roy S, Laerum F, Brosstad F, et al. Animal model of acute deep vein thrombosis. Cardiovasc Intervent Radiol 1998;21:329-33.

63. Hamer JD, Malone PC. Experimental deep venous thrombogenesis by a non-invasive method. Ann R Coll Surg Engl 1984;66:416-9.

64. Monreal M, Silveira P, Monreal L, et al. Comparative study on the antithrombotic efficacy of four low-molecular-weight heparins in three different models of experimental venous thrombosis. Haemostasis 1991;21:91-7.

65. Wang J, Tan HQ, Li MH, et al. Development of a new model of transvenous thrombosis in the pig superior sagittal sinus using thrombin injection and balloon occlusion. J Neuroradiol 2010;37:109-15.

66. Minko P, Bucker A, Laschke M, et al. Mechanical thrombectomy of iliac vein thrombosis in a pig model using the Rotarex and Aspirex catheters. Cardiovasc Intervent Radiol 2014;37:211-7.

67. Robinson VJ, Pineda GE, Salah AK, et al. Latex D-dimer signal in in situ femoral vein thrombus in swine and effect of minidose exogenous tissue plasminogen activator bolus. Chest 2005;127:622-9.

68. Shi WY, Wu S, Hu LY, et al. Swine Model of Thrombotic Caval Occlusion Created by Autologous Thrombus Injection with Assistance of Intra-caval Net Knitting. Sci Rep 2015;5:18546.

69. Gailani D, Cheng Q, Ivanov IS. Murine models in the evaluation of heparan sulfate-based anticoagulants. Methods Mol Biol 2015;1229:483-96.

70. Westrick RJ, Winn ME, Eitzman DT. Murine models of vascular thrombosis (Eitzman series). Arterioscler Thromb Vasc Biol 2007;27:2079-93.

71. Jagadeeswaran P, Cooley BC, Gross PL, et al. Animal Models of Thrombosis From Zebrafish to Nonhuman Primates: Use in the Elucidation of New Pathologic Pathways and the Development of Antithrombotic Drugs. Circ Res 2016;118:1363-79.

Cite this article as: Albadawi $\mathrm{H}$, Witting AA, Pershad $\mathrm{Y}$, Wallace A, Fleck AR, Hoang P, Khademhosseini A, Oklu R. Animal models of venous thrombosis. Cardiovasc Diagn Ther 2017;7(Suppl 3):S197-S206. doi: 10.21037/cdt.2017.08.10 